Ana Paula Mastropietro'

Érika Arantes de Oliveira"

Manoel Antônio dos Santos"

Júlio César Voltarelli'

\section{Functional Assessment of Cancer Therapy Bone Marrow Transplantation: tradução e validação}

\section{Functional Assessment of Cancer Therapy Bone Marrow Transplantation: Portuguese translation and validation}

\section{RESUMO}

OBJETIVO: Traduzir para o português e validar o questionário de qualidade de vida Functional Assessment of Cancer Therapy - Bone Marrow Transplantation (FACT-BMT) em pacientes transplantados de medula óssea.

MÉTODOS: O estudo foi realizado em Ribeirão Preto, SP, em 2005. O FACTBMT (versão 3) traduzido e a versão em português do Short Form-36 Health Survey (SF-36) foram aplicados simultaneamente em 55 pacientes consecutivos com leucemia, submetidos ao transplante e em seguimento. Dois parâmetros clínicos foram utilizados para testar a sensibilidade do questionário: tempo decorrido do transplante e presença ou não de doença do enxerto contra o hospedeiro. Foi utilizada a análise de variância (ANOVA) com o teste post hoc de Tukey. Aplicou-se o coeficiente alfa de Cronbach, padronizado para todas as questões, escore final e domínios.

RESULTADOS: A média de idade dos pacientes foi $34,8 \pm 8,1$ anos, com escolaridade média de $10,8 \pm 4,7$ anos, sendo $78,1 \%$ do sexo feminino. A duração média de tempo pós-transplante foi de 29,8ะ32,19 meses. Nenhuma alteração do formato original do questionário foi observada no final do processo de tradução e adaptação cultural. A consistência interna foi alta $(0,88)$. A correlação entre o questionário traduzido e o SF-36 variou de 0,35 a 0,57, considerada de moderada a boa para a maioria dos domínios de qualidade de vida. A avaliação das validades de construto e concorrente foi satisfatória e estatisticamente significativa.

CONCLUSÕES: A versão para o português do FACT-BMT foi validada satisfatoriamente para a aplicação em pacientes brasileiros de ambos os sexos submetidos ao transplante de medula óssea.

DESCRITORES: Transplante de medula óssea, reabilitação. Qualidade de vida. Questionários. Tradução (produto). Validade dos testes. Reprodutibilidade dos testes.

\section{ABSTRACT}

OBJECTIVE: To translate into Portuguese and validate the "Functional Assessment of Cancer Therapy - Bone Marrow Transplantation” (FACT-BMT) 
quality-of-life questionnaire, among bone marrow transplantation patients.

METHODS: The study was carried out in Ribeirão Preto, Southeastern Brazil in 2005. After translating FACT-BMT (version 3) into Portuguese, it was applied to 55 consecutive leukemia patients simultaneously with the Portuguese version of the Short Form-36 Health Survey (SF-36). These patients had undergone transplantation and were being followed up. Two clinical parameters were used for testing the sensitivity of the questionnaire: time elapsed since transplantation and presence or absence of graft-versushost disease. Analysis of variance with the post-hoc Tukey test was used. Cronbach's alpha coefficient was applied, standardized for all the questions, final scores and domains.

RESULTS: The patients' mean age was $34.8 \pm 8.1$ years and mean schooling was $10.8 \pm 4.7$ years, and $78.1 \%$ of the patients were female. The mean time since transplantation was $29.8 \pm 32.19$ months. At the end of the translation and cultural adaptation process, it was seen that there had not been any alteration to the original format of the questionnaire. The internal consistency was high (0.88). The correlation between the translated questionnaire and SF-36 ranged from 0.35 to 0.57 and was considered to be moderate to good for most quality-of-life domains. The evaluation of the construct and concurrent validities was satisfactory and statistically significant.

CONCLUSIONS: The Portuguese version of FACT-BMT was satisfactorily validated for application to Brazilian patients of both sexes undergoing bone marrow transplantation.

KEYWORDS: Bone marrow transplantation, rehabilitation. Quality of life. Questionnaires. Translation (product). Validity of tests. Reproducibility of tests.

\section{INTRODUÇÃO}

O transplante de medula óssea (TMO) é um procedimento de alta complexidade, cujo desenvolvimento, nas últimas décadas, permitiu o tratamento de doenças que antes eram invariavelmente fatais. ${ }^{1}$

Diversos estudos sobre qualidade de vida de pacientes submetidos ao TMO ressaltam a necessidade de correlacionar as variáveis clínicas, como tempo pós-TMO e existência de doença do enxerto contra o hospedeiro $(\mathrm{DECH})$, e variáveis demográficas, como sexo e idade. $., 8,10,11$

De acordo com a literatura, existem vários instrumentos ou índices utilizados para avaliar e mensurar a qualidade de vida de pacientes submetidos ao TMO, divididos em genéricos e específicos. Ambos fornecem informações complementares, de modo que podem ser empregados concomitantemente. ${ }^{13}$

Nessa perspectiva, autores ${ }^{13}$ salientam a necessidade de que a avaliação da qualidade de vida seja realizada por meio de um instrumento sensível, específico e de fácil reprodução. É fundamental que ele seja adaptado à condição cultural, e validado para o idioma do país onde será utilizado. ${ }^{3}$
No Brasil foram realizados estudos que utilizaram o questionário Short Form-36 Health Survey (SF-36), instrumento geral para a avaliação de qualidade de vida por meio de 36 questões, validado no Brasil por Ciconelli et al $^{6}$ em 1999. No País não há questionários de qualidade de vida específicos para TMO, o que justifica a condução de um estudo de validação de instrumento com essa finalidade.

Entre os diversos questionários específicos disponíveis para a avaliação da qualidade de vida de pacientes submetidos ao TMO destaca-se o Functional Assessment of Cancer Therapy - Bone Marrow Transplantation (FACT-BMT), que inclui aspectos funcionais e variáveis de qualidade de vida. ${ }^{9,11,12,16,18}$ O FACT-BMT é um questionário simples, breve e auto-administrável, originalmente desenvolvido e validado na língua inglesa por McQuellon et al. ${ }^{15}$

O objetivo do presente estudo foi traduzir o Functional Assessment of Cancer Therapy - Bone Marrow Transplantation (versão 3) para a língua portuguesa, considerando sua adaptação cultural e validação para o uso em pesquisas no Brasil. 


\section{MÉTODOS}

Foi realizado estudo observacional, tipo corte transversal, com 55 pacientes consecutivos, de ambos os sexos, que estiveram em seguimento no ambulatório da unidade de TMO de um hospital universitário de Ribeirão Preto, no período de novembro de 2004 a junho de 2005. Foram incluídos pacientes com idade superior a 21 anos; que tivessem pelo menos três anos de escolaridade formal; com disponibilidade para colaborar voluntariamente com a pesquisa; que fizeram o TMO nesse hospital e que estavam em acompanhamento, atendidos no hospital-dia e ambulatório no período da pesquisa. Foram excluídos portadores de transtornos psiquiátricos e doenças mentais, nos quais o juízo crítico da realidade e/ou capacidade cognitiva estivesse(m) comprometidos, prejudicando a aplicação e entendimento dos instrumentos.

Em um único encontro foram coletados os dados: sociodemográficos idade (até 30 anos e acima de 30 anos), sexo, escolaridade (até oito anos e acima de oito anos), estado civil, renda familiar (até dois salários mínimos e acima de dois salários mínimos); e variáveis clínicas tempo de TMO ( $<1$ ano - G1, de 1 a 2 anos - G2, >4 anos - G3) e diagnóstico de DECH (sim ou não).

O FACT-BMT (versão 3) é composto de 47 questões, sendo 41 delas utilizadas para obtenção do escore; as seis questões restantes são apresentadas por fornecer informações de síntese, porém não devem ser utilizadas para a obtenção do escore final. As questões são distribuídas em seis domínios. O FACT-BMT é pontuado pelos seus domínios, pela soma dos escores de suas questões. $\mathrm{O}$ formato Likert das respostas permite escores de zero a quatro para cada questão, sendo considerado o escore reverso para as questões construídas de forma negativa. O escore final do FACT-BMT varia de zero a 164 .

Duas traduções foram feitas por brasileiros, fluentes na língua inglesa e cientes dos objetivos de pesquisa. Após harmonização de ambas, a tradução denominada versão 1 foi retrovertida para o inglês por duas pessoas nativas nos Estados Unidos, fluentes em português e não cientes dos objetivos da pesquisa. Após harmonização da retroversão denominada versão 2 com a original em inglês, a tradução em língua portuguesa versão 1 foi considerada gramatical e semanticamente equivalente à versão original em inglês e apta para ser submetida a um comitê de cinco juízes bilíngües, todos profissionais relacionados à área da saúde. Após serem consideradas suas sugestões, a versão em português do FACT-BMT versão 1 foi pré-testada em 27 pacientes submetidos ao TMO. ${ }^{14}$

A única alteração da tradução em relação ao texto original ocorreu na questão número 44: "I have concerns about my ability to have children”. A tradução inicial foi: "Tenho consciência da minha capacidade de ter filhos". A versão em português foi considerada gramatical e semanticamente correta, com base na tradução e retrotradução. Porém, durante a fase de pré-teste, observou-se dificuldade de compreensão pela maioria dos pacientes. Por esse motivo, a questão foi modificada para: "Estou preocupado(a) com a minha capacidade de ter filhos". Com essa frase, no segundo pré-teste, obteve-se taxa de compreensão de $91 \%$. Assim, a versão final do FACT-BMT foi considerada finalizada para ser aplicada na amostra de 55 pacientes.

Os pacientes foram solicitados a preencher as versões na língua portuguesa dos questionários FACT-BMT e SF-36, alternando-se a ordem de cada instrumento de modo a controlar os efeitos da apresentação. O questionário SF-36 é composto por 36 questões, distribuídas em sete domínios: capacidade funcional, aspectos físicos, dor, estado geral da saúde, vitalidade, aspectos emocionais e saúde mental. Apresenta o escore final de zero a cem, no qual zero corresponde ao pior estado geral de saúde e cem ao melhor estado de saúde.

A validade de construto foi avaliada pela comparação da versão validada em português do SF-36 com a versão em português do FACT-BMT. A validade concorrente foi avaliada determinando-se a capacidade de o FACT-BMT discriminar subgrupos de pacientes com diferentes tempos pós-TMO, e com ou sem diagnóstico de DECH.

A análise descritiva foi realizada por meio da freqüência das variáveis categóricas e medidas de posição central e de dispersão das variáveis contínuas.

Como medida de confiabilidade foram avaliadas a consistência interna do instrumento e o coeficiente de correlação. Para estimar a consistência interna aplicouse o coeficiente alfa de Cronbach, padronizado para todas as questões, escore final e domínios. Para verificar a correlação entre todos os domínios do FACT-BMT e o escore final foi utilizado o coeficiente de correlação não-paramétrico de Spearman.

Para a comparação dos domínios do FACT-BMT entre os diferentes níveis da variável tempo pós-TMO foi utilizada a análise de variância (ANOVA) com o teste post hoc de Tukey. A normalidade das variáveis foi testada pelo método de Kolmogorov-Smirnov.

Para a comparação dos domínios da FACT-BMT entre os níveis da variável DECH foi utilizado o teste t para as amostras independentes.

Para o estudo das correlações entre as variáveis (domínios) do FACT-BMT com as variáveis (domínios) do SF-36 e entre si, foi utilizado o coeficiente de correlação não-paramétrico de Spearman. Os cálculos foram efetuados no programa SPSS 10.0. 
O projeto foi aprovado pelo Comitê de Ética em Pesquisa do Hospital das Clínicas da Faculdade de Medicina de Ribeirão Preto da Universidade de São Paulo, processo HCRP $n^{\circ}$ 10700/2004. Os pacientes assinaram o termo de consentimento livre e esclarecido.

\section{RESULTADOS}

Os 55 pacientes estudados tinham as seguintes características: média de idade de 34,8 $\pm 8,1$ anos, escolaridade média de $10,8 \pm 4,7$ anos, $78,1 \%$ do sexo feminino e duração média de tempo pós-TMO de 29,8 332,2 meses.

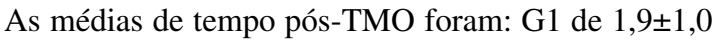

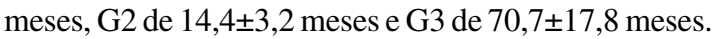
Em relação à presença de $\mathrm{DECH}, 34,5 \%$ dos pacientes receberam o diagnóstico.

O estudo das associações entre os escores dos questionários analisados e as categorias das variáveis sociodemográficas indicou diferenças significativas nas correlações com os domínios da FACT-BMT. A correlação das variáveis escolaridade e renda familiar

Tabela 1. Análise de consistência interna dos domínios do Functional Assessment of Cancer Therapy Bone Marrow Transplantation aplicado em pacientes transplantados de medula óssea. Ribeirão Preto, SP, 2005. (N=55)

\begin{tabular}{lc}
\hline Domínio & $\begin{array}{c}\text { Coeficiente } \\
\text { Alfa de Cronbach }\end{array}$ \\
\hline Bem-estar físico & 0,73 \\
Bem-estar social/familiar & 0,70 \\
Relacionamento com o médico & 0,80 \\
Bem-estar emocional & 0,70 \\
Bem-estar funcional & 0,78 \\
Preocupações adicionais & 0,65 \\
\hline Total & 0,88 \\
\hline
\end{tabular}

$(\mathrm{p}<0,0001)$ indicou que pacientes com escolaridade acima de oito anos e renda familiar acima de dois salários mínimos tiveram maiores escores de qualidade de vida; a correlação da variável sexo e os domínios preocupações adicionais $(\mathrm{p}=0,02)$, aspectos sociais $(p=0,02)$ e dor $(p<0,0001)$, mostrou que os homens apresentaram escores maiores do que as mulheres; finalmente quanto à correlação da variável idade e aspectos sociais $(\mathrm{p}=0,04)$, observou-se que os adultos obtiveram maiores escores do que os jovens.

O alfa de Cronbach, utilizado para avaliar a acurácia do instrumento foi satisfatório, com valor de 0,88 ; seus valores para os domínios variaram de 0,65 a 0,88 , sendo o valor menor gerado pelo item 44. Com a exclusão dessa questão, verificou-se pequena elevação do valor de alfa, para 0,70 , sugerindo fraca correlação dessa questão com o domínio preocupações adicionais (Tabela 1).

Os coeficientes de correlação de consistência interna variaram de 0,29 a 0,88 , tendo todos os domínios correlações significativas entre si $(\mathrm{p}<0,0001)$. As melhores correlações ocorreram, principalmente, entre bem-estar funcional e escore total $(0,88)$ e entre preocupações adicionais e escore total $(0,80)$ (Tabela 2$)$.

A validade de construto foi analisada pela medida das correlações entre os domínios do FACT-BMT e os domínios do SF-36. Observou-se correlações significativas entre o escore final do FACT-BMT e todos os domínios do SF-36 (p<0,0001). O domínio da "saúde mental" e "ausência de dor" apresentaram menor correlação $(0,35)$. A maior correlação aconteceu entre os domínios "estado geral de saúde" e "aspecto físico", cujo coeficiente foi 0,52 . A validade concorrente foi avaliada pela correlação entre algumas variáveis sociodemográficas e clínicas com os domínios e escore final do questionário FACT-BMT.

Todas as médias e desvios-padrão mostraram que os pacientes com menos tempo de TMO apresentam

Tabela 2. Coeficiente de correlação de Spearman entre os domínios do Functional Assessment of Cancer Therapy Bone Marrow Transplantation aplicado em transplantados de medula óssea. Ribeirão Preto, SP, 2005. (N=55)

\begin{tabular}{|c|c|c|c|c|c|c|}
\hline Domínio & $\begin{array}{l}\text { Bem-estar } \\
\text { físico }\end{array}$ & $\begin{array}{c}\text { Bem-estar social/ } \\
\text { familiar }\end{array}$ & $\begin{array}{l}\text { Relacionamento } \\
\text { com o médico }\end{array}$ & $\begin{array}{l}\text { Bem-estar } \\
\text { emocional }\end{array}$ & $\begin{array}{l}\text { Bem-estar } \\
\text { funcional }\end{array}$ & $\begin{array}{l}\text { Preocupações } \\
\text { adicionais }\end{array}$ \\
\hline Bem-estar social/familiar & $\begin{array}{l}0,29 \\
0,03\end{array}$ & - & - & - & - & - \\
\hline $\begin{array}{l}\text { Relacionamento } \\
\text { com o médico }\end{array}$ & $\begin{array}{c}0,39 \\
0,003\end{array}$ & $\begin{array}{c}0,47 \\
<0,001\end{array}$ & $\begin{array}{l}- \\
-\end{array}$ & $\begin{array}{l}- \\
-\end{array}$ & $\begin{array}{l}- \\
-\end{array}$ & - \\
\hline Bem-estar emocional & $\begin{array}{c}0,55 \\
<0,001\end{array}$ & $\begin{array}{c}0,50 \\
<0,001\end{array}$ & $\begin{array}{l}0,44 \\
0,001\end{array}$ & $\begin{array}{l}- \\
-\end{array}$ & $\begin{array}{l}- \\
-\end{array}$ & $\begin{array}{l}- \\
-\end{array}$ \\
\hline Bem-estar funcional & $\begin{array}{c}0,56 \\
<0,001\end{array}$ & $\begin{array}{c}0,62 \\
<0,001\end{array}$ & $\begin{array}{c}0,53 \\
<0,001\end{array}$ & $\begin{array}{c}0,67 \\
<0,001\end{array}$ & - & - \\
\hline Preocupações adicionais & $\begin{array}{c}0,58 \\
<0,001\end{array}$ & $\begin{array}{l}0,34 \\
0,01\end{array}$ & $\begin{array}{c}0,40 \\
0,003\end{array}$ & $\begin{array}{c}0,54 \\
<0,001\end{array}$ & $\begin{array}{c}0,65 \\
<0,001\end{array}$ & $\begin{array}{l}- \\
-\end{array}$ \\
\hline Total & $\begin{array}{c}0,69 \\
<0,001\end{array}$ & $\begin{array}{c}0,70 \\
<0,001\end{array}$ & $\begin{array}{c}0,63 \\
<0,001\end{array}$ & $\begin{array}{c}0,79 \\
<0,001\end{array}$ & $\begin{array}{c}0,88 \\
<0,001\end{array}$ & $\begin{array}{c}0,80 \\
<0,001\end{array}$ \\
\hline
\end{tabular}


Tabela 3. Análise de variância dos domínios do Functional Assessment of Cancer Therapy Bone Marrow Transplantation em função do tempo pós-transplante da medula óssea. Ribeirão Preto, SP, 2005. (N=55)

\begin{tabular}{lcccc}
\hline \multirow{2}{*}{ Domínio } & G1 & G2 & G3 & p \\
\hline Bem-estar físico & $20,05 \pm 4,07$ & $23,38 \pm 3,79$ & $25,15 \pm 2,43$ & $<0,001$ \\
Bem-estar social/familiar & $18,00 \pm 6,32$ & $20,00 \pm 4,99$ & $21,15 \pm 4,59$ & 0,20 \\
Relacionamento com o médico & $6,61 \pm 1,53$ & $6,72 \pm 1,22$ & $7,47 \pm 0,90$ & 0,05 \\
Bem-estar emocional & $19,83 \pm 2,74$ & $20,27 \pm 3,47$ & $21,36 \pm 3,46$ & 0,34 \\
Bem-estar funcional & $14,38 \pm 3,82$ & $19,11 \pm 4,18$ & $22,47 \pm 4,32$ & $<0,001$ \\
Preocupações adicionais & $29,22 \pm 5,07$ & $32,72 \pm 6,08$ & $37,78 \pm 5,52$ & $<0,001$ \\
\hline Total & $108,11 \pm 17,50$ & $122,22 \pm 15,64$ & $135,42 \pm 16,45$ & $<0,001$ \\
\hline
\end{tabular}

G1: menos de 1 ano pós-transplante de medula óssea

G2: de 1 a 2 anos pós-transplante de medula óssea

G3: acima de 4 anos pós-transplante de medula óssea

Tabela 4. Análise de variância dos domínios do Functional Assessment of Cancer Therapy Bone Marrow Transplantation em função do diagnóstico da doença do enxerto contra o hospedeiro (DECH). Ribeirão Preto, SP, 2005. (N=55)

\begin{tabular}{|c|c|c|c|}
\hline Domínio & $\begin{array}{l}\text { Com DECH } \\
(\mathrm{N}=19) \\
\text { Média } \pm \mathrm{DP}\end{array}$ & $\begin{array}{l}\text { Sem DECH } \\
(\mathrm{N}=36) \\
\text { Média } \pm \mathrm{DP}\end{array}$ & $\mathrm{p}$ \\
\hline Bem-estar físico & $20,78 \pm 4,18$ & $24,02 \pm 3,52$ & $<0,001$ \\
\hline Bem-estar social/familiar & $14,84 \pm 3,96$ & $22,33 \pm 4,13$ & $<0,001$ \\
\hline Relacionamento com o médico & $7,41 \pm 0,87$ & $6,05 \pm 1,47$ & $<0,001$ \\
\hline Bem-estar emocional & $17,73 \pm 3,70$ & $21,97 \pm 1,71$ & $<0,001$ \\
\hline Bem-estar funcional & $14,57 \pm 3,74$ & $20,91 \pm 4,59$ & $<0,001$ \\
\hline Preocupações adicionais & $28,94 \pm 5,20$ & $35,63 \pm 6,00$ & $<0,001$ \\
\hline Total & $102,94 \pm 12,27$ & $132,30 \pm 14,84$ & $<0,001$ \\
\hline
\end{tabular}

escores menores do que os pacientes com um a dois anos de TMO. Além disso, os pacientes com um a dois anos de TMO estavam piores do que aqueles que com mais de quatro anos de TMO. Essa diferença também pode ser observada pelo valor significativo $(\mathrm{p}<0,001)$ na maioria dos domínios (Tabela 3).

Ter diagnóstico de DECH interferiu negativamente na qualidade de vida da amostra $(\mathrm{p}<0,0001)$. Observou-se que em todos os domínios houve diferença estatística entre os grupos sem e com DECH (Tabela 4).

\section{DISCUSSÃO}

A falta de instrumentos de avaliação de qualidade de vida traduzidos e validados para o português na área de transplante de medula óssea tem restringido as pesquisas nesse campo no Brasil. Nesse segmento clínico, a versão em português do SF-36 provou ser confiável pela análise de suas propriedades de medida. ${ }^{6}$ Seu construto, entretanto, foi elaborado para avaliar qualidade de vida genérica na saúde. A decisão pelo desenvolvimento da tradução, adaptação cultural e validação do FACT-BMT deve-se ao fato de ser um instrumento que avalia especificamente o impacto do TMO na vida dos pacientes.

Uma das limitações do estudo relaciona-se ao nível de escolaridade da amostra populacional. Apesar de ser auto-aplicável, o questionário teve de ser lido pelo entrevistador para $13(23,6 \%)$ pacientes estudados. Esse recurso é de uso comum para a inclusão de pacientes com baixa ou nenhuma escolaridade. ${ }^{6}$

Os resultados sobre tempo pós-TMO e sua relação direta com qualidade de vida foram similares aos encontrados nos trabalhos internacionais. ${ }^{4,5,9,11,18,19}$ Tal achado pode ser explicado pela esperada melhora gradual da condição orgânica do paciente e pela redução das limitações decorrentes do próprio tratamento, como a impossibilidade de exercer atividades que envolvam esforços físicos, uso regular de medicações, retornos ambulatoriais freqüentes, possibilidade de recorrência da doença, dentre outras.

Explicação para a pior qualidade de vida de portadores de DECH, em sua forma aguda ou crônica, seria a necessidade de voltar ao tratamento com drogas imunossupressoras, estendendo assim as limitações. Esse dado corrobora os resultados de estudos realizados em outros países..$^{5,8}$ 
Os resultados do presente trabalho que indicaram melhor qualidade de vida entre os pacientes com melhor renda e escolaridade são consistentes com os da literatura internacional. ${ }^{2,17}$

A consistência interna do FACT-BMT, medida pela correlação dos domínios com suas questões, foi satisfatória, obtendo-se índice geral de 0,88 , e a consistência interna de seus domínios variou de 0,65 a 0,88 .

Comparando esses resultados com o do SF-36 e o da FACT-BMT na língua inglesa, tem-se que o SF-36 apresentou os coeficientes de correlação variando entre 0,1481 a 0,6189 , demonstrando uma correlação moderada entre eles. ${ }^{7}$ Já o alfa de Cronbach encontrado para a FACT-BMT na língua inglesa situa-se em um intervalo de 0,85 a $0,92 .{ }^{15}$

As hipóteses levantadas previamente foram confirmadas, pois pior qualidade de vida medida pelo FACT-

\section{REFERÊNCIAS}

1. Andrykowski MA. Psychosocial factors in bone marrow transplantation: a review and recommendations for research. Bone Marrow Transplant. 1994;13:357-75.

2. Andrykowski MA, Greiner CB, Altmaier EM, Burish TG, Antin JH, Gingrich R, et al. Quality of life following bone marrow transplantation findings from a multicentre study. Br J Cancer. 1995;71:1322-9.

3. Bonomi A, Cella DF, Hahn EA, Bjordal K, SpernerUnterweger B, Gangeri L, et al. Multilingual translation of the Functional Assessment of Cancer Therapy (FACT) quality of life measurement system. Qual Life Res. 1996;5:309-20.

4. Broers S, Hengeveld MW, Kaptein ADA, Cessie SLE, Loo FV, Vries T. Are pretransplant psychological variables related to survival after bone marrow transplantation?: a prospective study of 123 consecutive patients. J Psychosom Res. 1998;45(4):341-51.

5. Chiodi S, Spinelli S, Ravera G, Petti AR, Van Lint MT, Lamparelli T, et al. Quality of life in 244 recipients of allogeneic bone marrow transplantation. $\mathrm{Br} /$ Haematol. 2000;110:614-9.

6. Ciconelli RM, Ferraz MB, Santos W, Meinão I, Quaresma MR. Tradução para a língua portuguesa e validação do questionário genérico de avaliação da qualidade de vida da SF-36 (Brasil SF-36). Rev Bras Reumatol. 1999;39(3):143-50.

7. Ciconelli RM. Medidas de avaliação de qualidade de vida. Rev Bras Reumatol. 2003;43:9-13.

8. Deeg HJ, Leisenring W, Storb R, Nims J, Flowers ME, Witherspoon RP, et al. Long-term outcome after bone marrow transplantation for severe plastic anemia. Blood. 1998;91(10):3637-45.

9. Heinomen $\mathrm{H}$, Volin L, Uutela A, Zevon $\mathrm{M}$, Barrick C, Ruutu T. Quality of life and factors related to
BMT esteve relacionada aos parâmetros clínicos e sociodemográficos pesquisados. Dessa forma obteve-se que os maiores índices de qualidade de vida apareceram nos pacientes com escolaridade acima de oito anos, renda familiar acima de dois salários, no sexo masculino, adultos e sem DECH.

Concluindo, a versão do FACT-BMT foi traduzida e validada com sucesso para o português (Anexo), de acordo com o resultado da análise final de suas propriedades de medida. Simplicidade e brevidade de aplicação tornam esse questionário um instrumento prático e disponível para utilização em pesquisas clínicas no Brasil.

Atualmente o FACT-BMT encontra-se traduzido para o português na versão 4 , ainda não validada, e que pode ser obtida por intermédio da Functional Assessment of Chronic Illness Therapy (FACIT) Multilingual Translations Project. perceived satisfaction with quality of life allogeneic bone marrow transplantation. Ann Hematol. 2001;80:137-43.

10. Heinomen H, Volin L, Uutela A, Zevon M, Barrick C, Ruutu T. Gender-associated differences in the quality of life after allogeneic BMT. Bone Marrow Transplant. 2001;28(5):503-9.

11. Koop M, Schweigkofler H, Holzner B, Nachbaur D, Niederwieser D, Fleischhacker WW, et al. Time after bone marrow transplantation as an important variable for quality of life: results of a cross-sectional investigation using two different instruments for qualityof-life assessment. Ann Hematol. 1998;77:27-32.

12. Kopp M, Schweigkofler H, Holzner B, Nachbaur D, Niederwieser D, Fleischhacker WW, et al. EORTC QLQ-C30 and FACT-BMT for the measurement of quality of life in bone marrow transplant recipients: a comparison. Eur J Haematol. 2000;65:97-103.

13. Linde C. How evaluate quality of life in pacemaker patients: problems and pitfalls. Pace. 1996;19(Part 1):391-7.

14. Mastropietro AP, Oliveira EA, Santos MA. Reinserção profissional de pacientes submetidos ao transplante de medula óssea. Rev Centro Univ Claretiano Batatais. 2003;3:139-45.

15. Mcquellon RP, Russell GB, Cella DF, Craven BL, Brady $\mathrm{M}$, Bonomi A, et al. Quality of life measurement in bone marrow transplantation: development of Functional Assessment of Cancer Therapy - Bone Marrow Transplant (FACT-BMT) scale. Bone Marrow Transplant. 1997;19:357-68.

16. Mcquellon RP, Russell GB, Rambo TD, Craven BL, Radford J, Perry JJ, et al. Quality of life and psychological distress of bone marrow transplant recipients: the time trajectory's recovery over the first year. Bone Marrow Transplant. 1998;21:477-86. 
17. Prieto JM, Saez R, Carreras E, Atala J, Sierra J, Rovira $M$, et al. Physical and psychosocial functioning of 117 survivors of bone marrow transplantation. Bone Marrow Transplant. 1996;17:1133-42.

18. Saleh US, Brockopp DY. Quality of life one year following bone marrow transplantation: psychometric evaluation of the quality of life in bone marrow transplant survivor's tool. Oncol Nurs Forum. 2001;28(9):1457-64.

19. Sutherland HJ, Fyles GM, Adams G, Hao Y, Lipton $\mathrm{JH}$, Minden MD, et al. Quality of life following bone marrow transplantation: a comparison of patient report with population norms. Bone Marrow Transplant. 1997; 19:1129-36. 


\section{ANEXO}

\section{Functional Assessment Cancer Therapy - Bone Marrow Transplantation (Versão 3)}

NOME:

LOCAL DE APLICAÇÃO:

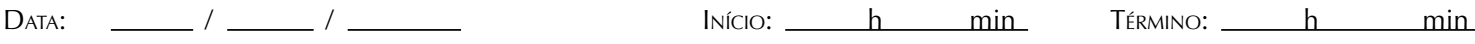
Abaixo você encontrará uma lista de declarações, que outras pessoas com a sua doença disseram ser importantes. Fazendo um círculo em um número por linha, favor indicar até que ponto cada declaração foi verdadeira para você durante os últimos sete dias.

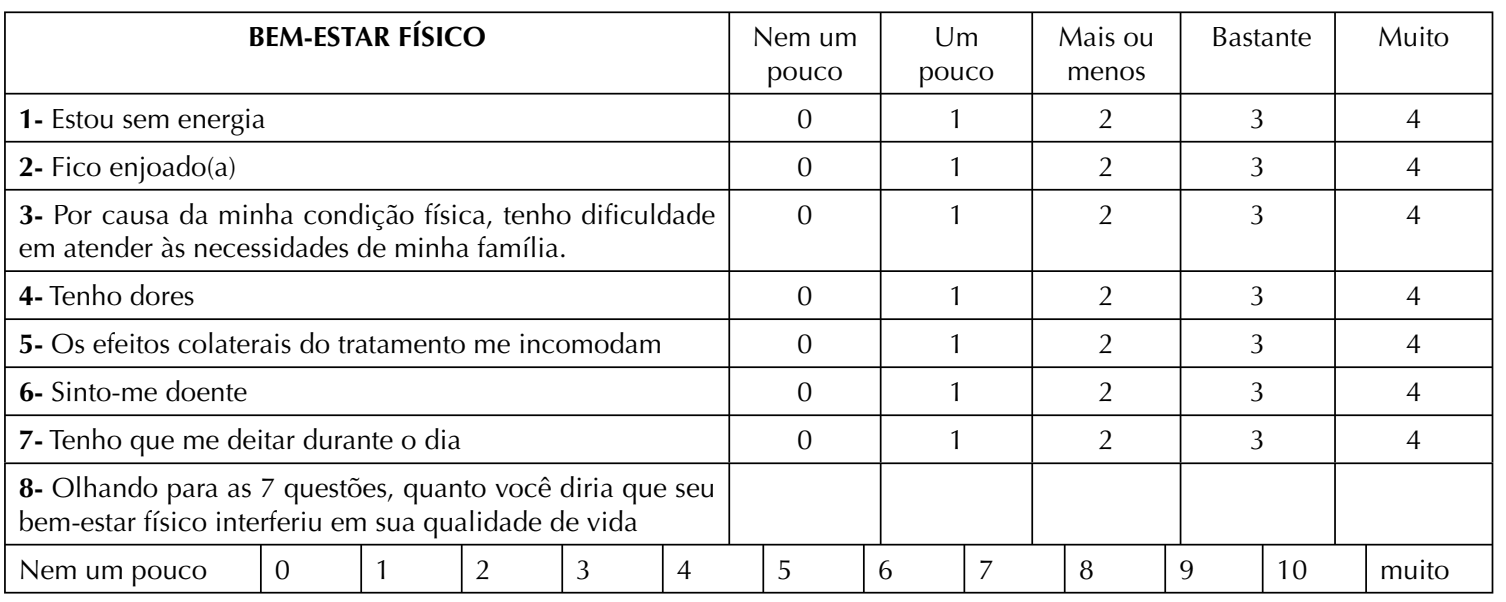

\begin{tabular}{|c|c|c|c|c|c|c|c|c|c|c|}
\hline \multicolumn{6}{|c|}{ BEM-ESTAR SOCIAL / FAMILIAR } & $\begin{array}{l}\text { Nem um } \\
\text { pouco }\end{array}$ & $\begin{array}{l}\text { Um } \\
\text { pouco }\end{array}$ & $\begin{array}{c}\text { Mais ou } \\
\text { menos }\end{array}$ & Bastante & Muito \\
\hline \multicolumn{6}{|c|}{$\begin{array}{l}\text { 9- Sinto que tenho um relacionamento distante com os } \\
\text { meus amigos }\end{array}$} & 0 & 1 & 2 & 3 & 4 \\
\hline \multicolumn{6}{|c|}{ 10- Recebo apoio emocional da minha família } & 0 & 1 & 2 & 3 & 4 \\
\hline \multicolumn{6}{|c|}{ 11- Recebo apoio dos meus amigos } & 0 & 1 & 2 & 3 & 4 \\
\hline \multicolumn{6}{|c|}{ 12- A minha família aceita a minha doença } & 0 & 1 & 2 & 3 & 4 \\
\hline \multicolumn{6}{|c|}{$\begin{array}{l}\text { 13- Estou insatisfeito(a) com a comunicação da família sobre } \\
\text { a minha doença }\end{array}$} & 0 & 1 & 2 & 3 & 4 \\
\hline \multicolumn{6}{|c|}{$\begin{array}{l}\text { 14- Sinto-me próximo(a) do(a) meu(minha) parceiro(a) (ou } \\
\text { pessoa que me dá maior apoio) }\end{array}$} & 0 & 1 & 2 & 3 & 4 \\
\hline \multicolumn{6}{|c|}{$\begin{array}{l}\text { 15- Você teve relação sexual durante o ano passado? } \\
\text { NÃO__SIM__. Se SIM: Eu estou satisfeito com minha } \\
\text { vida sexual }\end{array}$} & 0 & 1 & 2 & 3 & 4 \\
\hline \multicolumn{6}{|c|}{$\begin{array}{l}\text { 16- Olhando para as } 7 \text { questões, quanto você diria que sua } \\
\text { vida social/familiar interferiu em sua qualidade de vida }\end{array}$} & & & & & \\
\hline Nem um pouco & 0 & 1 & 2 & 3 & 4 & 5 & 7 & 8 & 10 & muito \\
\hline
\end{tabular}

\begin{tabular}{|c|c|c|c|c|c|c|c|c|c|c|}
\hline \multicolumn{6}{|c|}{ RELACIONAMENTO COM O MÉDICO } & $\begin{array}{l}\text { Nem um } \\
\text { pouco }\end{array}$ & $\begin{array}{l}\text { Um } \\
\text { pouco }\end{array}$ & $\begin{array}{c}\text { Mais ou } \\
\text { menos }\end{array}$ & Bastante & Muito \\
\hline \multicolumn{6}{|c|}{ 17- Tenho confiança no(s) meu(s) médico(s) } & 0 & 1 & 2 & 3 & 4 \\
\hline \multicolumn{6}{|c|}{$\begin{array}{l}\text { 18- Meu(s) médico(s) é(são) capaz de responder minhas } \\
\text { questões }\end{array}$} & 0 & 1 & 2 & 3 & 4 \\
\hline \multicolumn{6}{|c|}{$\begin{array}{l}\text { 19- Olhando para as } 2 \text { questões, quanto você diria que seu } \\
\text { relacionamento com o médico interferiu em sua qualidade } \\
\text { de vida }\end{array}$} & & & & & \\
\hline Nem um pouco & 0 & 1 & 2 & 3 & 4 & 5 & 7 & 8 & 10 & muito \\
\hline
\end{tabular}




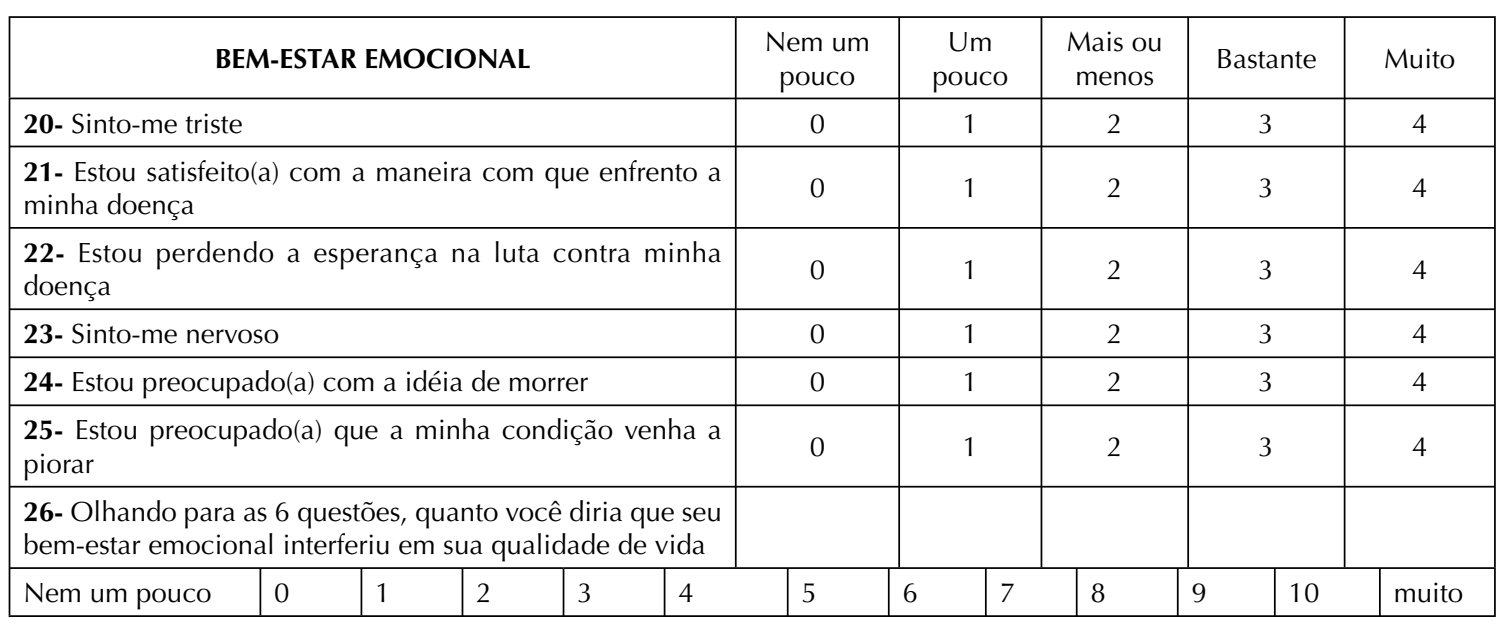

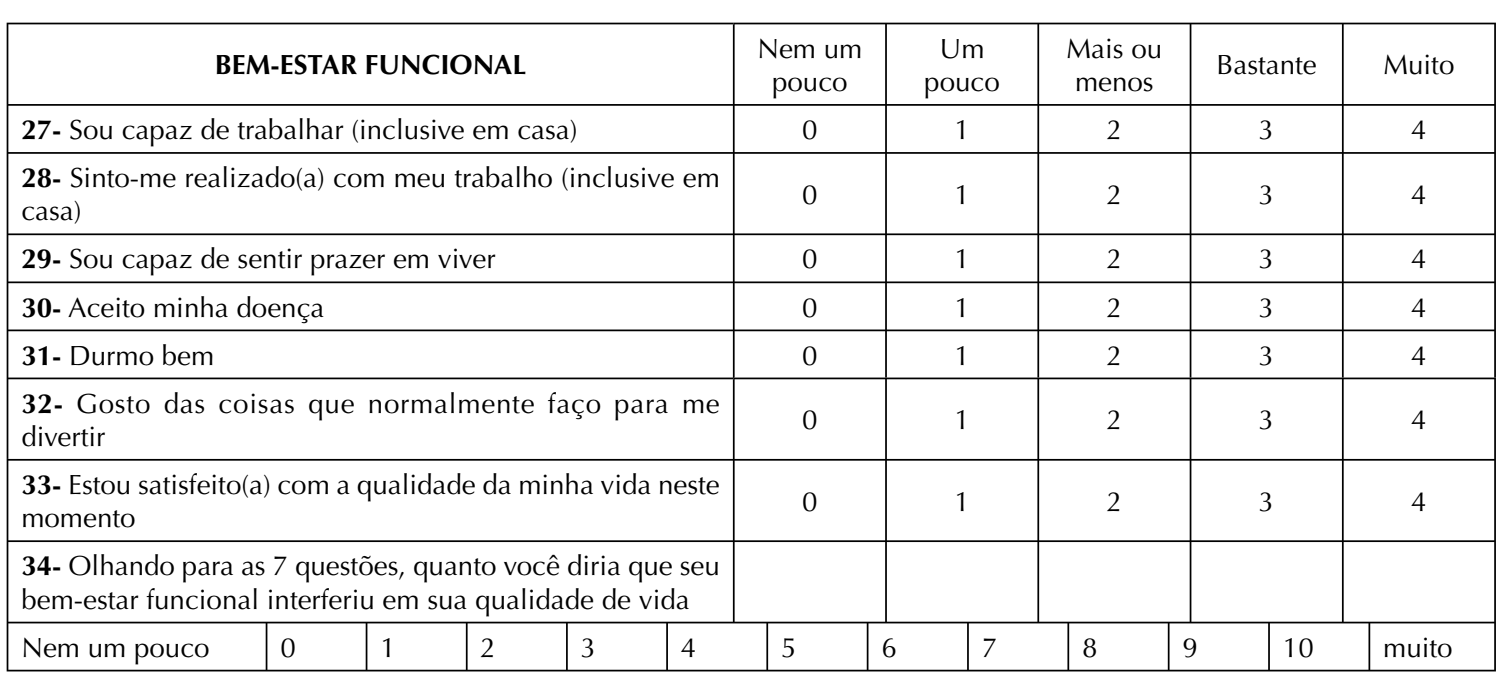

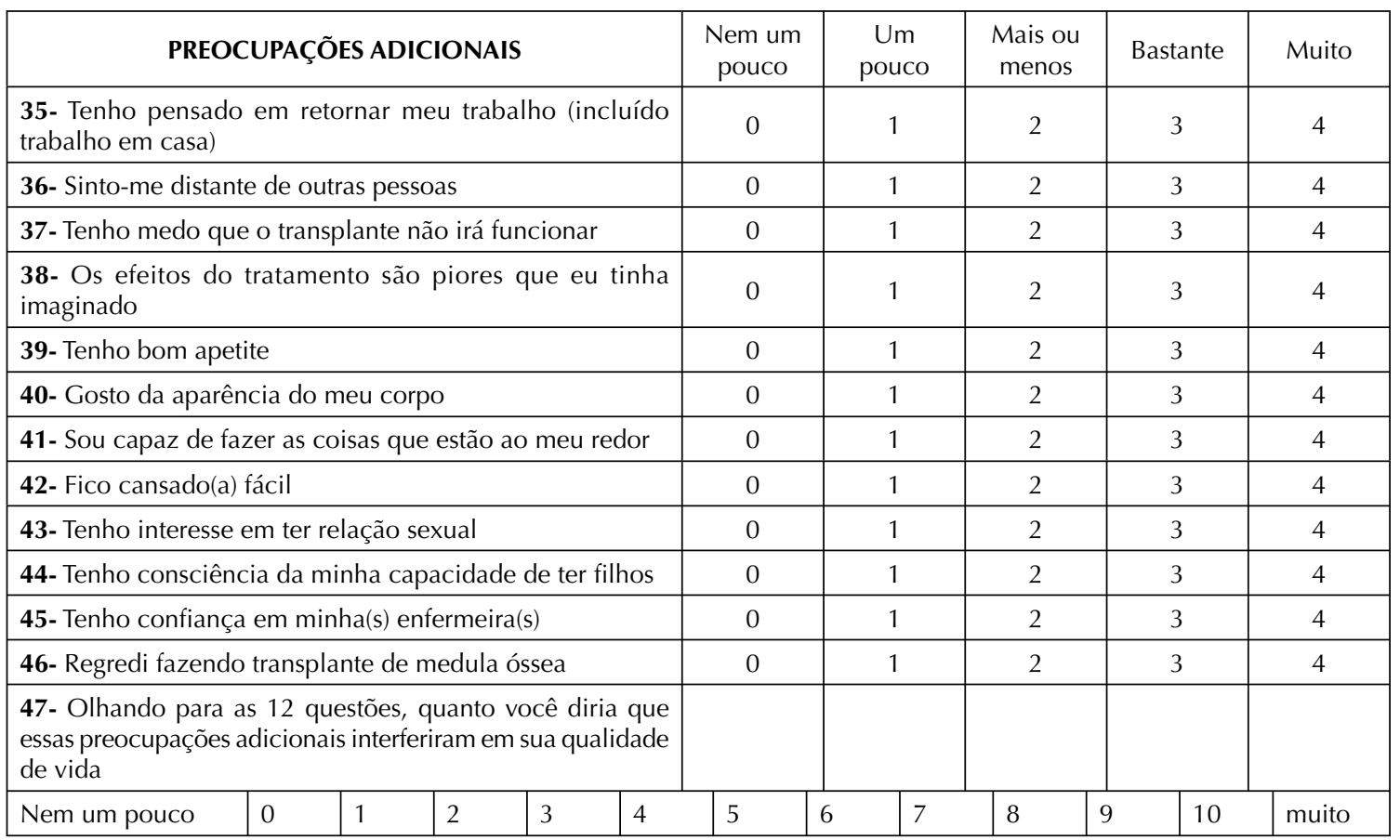

